\title{
Acompanhamento dos bebês de alto risco em serviços de saúde: compromisso a favor da vida
}

RESUMO | Objetivo: identificar os motivos que levam as mães de bebês de alto risco a abandonarem o acompanhamento no Ambulatório de Alto Risco, ao longo do primeiro ano de vida. Método: estudo descritivo e exploratório, de natureza qualitativa realizado com 16 mães de bebês que foram acompanhados no Ambulatório de Alto Risco vinculado ao Programa Rede Mãe Paranaense. A coleta de dados ocorreu entre os meses de agosto e setembro de 2020, mediante entrevistas semiestruturadas e submetidas à análise de conteúdo, modalidade temática. Resultados: Emergiram duas categorias: Abandono do acompanhamento infantil de alto risco: desconhecimento atrelado a falta de apoio social; Ansiedade frente ao desconhecido: vivência das mães após o nascimento. Conclusão: os motivos referentes aos aspectos maternos, familiares, sociais e características dos serviços de saúde podem influenciar no abandono do acompanhamento infantil.

Descritores: Saúde da criança; Serviços de Saúde Materno-Infantil; Desenvolvimento infantil; Pacientes Desistentes do Tratamento.

ABSTRACT | Objective: to identify the reasons that lead mothers of high-risk babies to abandon follow-up at the High-Risk Outpatient Clinic, throughout the first year of life. Method: descriptive and exploratory study, qualitative in nature, conducted with 16 mothers of babies who were followed at the High Risk Outpatient Clinic linked to the Rede Mãe Paranaense Program. Data collection took place between August and September 2020, through semi-structured interviews and submitted to content analysis, thematic modality. Results: Two categories emerged: Abandonment of high-risk child monitoring: lack of knowledge linked to lack of social support; Anxiety in the face of the unknown: mothers' experience after birth. Conclusion: the reasons related to maternal, family, social aspects and characteristics of health services can influence the abandonment of child care.

Descriptors: Child health; Maternal and Child Health Services; Child development; Treatment Dropout Patients;

RESUMEN | Objetivo: identificar los motivos que llevan a las madres de bebés de alto riesgo a abandonar el seguimiento en la Clínica Ambulatoria de Alto Riesgo, a lo largo del primer año de vida. Método: estudio descriptivo y exploratorio, de carácter cualitativo, realizado con 16 madres de bebés que fueron seguidos en la Clínica de Alto Riesgo vinculada al Programa Rede Mãe Paranaense. La recolección de datos se realizó entre agosto y septiembre de 2020, a través de entrevistas semiestructuradas y sometidas a análisis de contenido, modalidad temática. Resultados: Surgieron dos categorías: Abandono del monitoreo infantil de alto riesgo: desconocimiento vinculado a falta de apoyo social; Ansiedad ante lo desconocido: la experiencia de las madres después del nacimiento. Conclusión: las razones relacionadas con aspectos maternos, familiares, sociales y características de los servicios de salud pueden influir en el abandono del cuidado infantil.

Descriptores: Salud infantil; Servicios de salud maternoinfantil; Desarrollo infantil; Pacientes que abandonaron el tratamiento.

\section{Rafaela Yukari Itako Kubo}

Universidade Estadual de Maringá/UEM. Maringá, Paraná, Brasil. Enfermeira.

ORCID:

\section{Bianca Machado Cruz Shibukawa}

Universidade Estadual de Maringá/UEM. Maringá, Paraná, Brasil. Enfermeira. Doutora em Enfermagem.

ORCID:

\section{Camila Moraes Garollo Piran}

Universidade Estadual de Maringá/UEM. Maringá, Paraná, Brasil. Enfermeira. Mestranda do Programa de Pós-Graduação em Enfermagem.

ORCID:

\section{Beatriz Sousa da Fonseca}

Universidade Estadual de Maringá/UEM. Maringá, Paraná, Brasil. Enfermeira. Mestranda do Programa de Pós-Graduação em Enfermagem.

ORCID:

\section{Marcela Demitto Furtado}

Universidade Estadual de Maringá/UEM. Maringá, Paraná, Brasil. Enfermeira. Doutora em Enfermagem.

ORCID:

\section{Maria de Fátima Garcia Lopes Merino}

Universidade Estadual de Maringá/UEM. Maringá, Paraná, Brasil. Enfermeira. Doutora em Enfermagem. ORCID:

Recebido em: 30/09/2021

Aprovado em: 18/11/2021
INTRODUÇÃO

maioria dos óbitos na infância
concentra-se no primeiro ano de
vida, sobretudo no primeiro mês de vida. Há uma elevada influência das causas perinatais, como a prematuridade, o que evidencia a importância dos fatores ligados à gestação, ao parto e ao pós-parto, em geral preveníeis por meio de assistência à saúde de qualidade. Estes decorrem de uma combinação de fatores biológicos, sociais, culturais e de falhas do sistema de saúde (1).

Nas últimas décadas o mundo passou por diversos avanços, no entanto, a mortalidade infantil ainda é um tema que gera preocupação para a saúde pública. A taxa global de mortalidade de crianças menores de cinco anos de idade, reduziu de $93(92,95)$ em 1990 
e $76(75,78)$ em 2000 para $39(37,42)$ mortes por 1.000 nascidos vivos em 2018 - 59\% e 49\%, respectivamente (2).

O Brasil conseguiu reduzir a mortalidade infantil entre 1990 e 2015, uma das metas dos Objetivos de Desenvolvimento do Milênio (ODM). Entretanto, no ano de 2016, a taxa de mortalidade infantil apresentou um crescimento, fato que não acontecia há 26 anos. De acordo com o Instituto Brasileiro de Geografia e Estatística, a taxa de mortalidade em 2018 foi de 12,4 para cada 1.000 nascidos vivos. Embora a taxa de mortalidade tenha caído pela metade de 1990 para 2018, estas ainda são consideradas elevadas e milhares de crianças ainda continuam morrendo todos os dias (3).

Em regiões de maior desenvolvimento econômico, como a região Sul e Sudeste, as taxas de mortalidades são menores. Já na região Norte e Nordeste encontram-se as maiores taxas de mortalidade, enquanto na região Centro-Oeste as taxas são medianas (4).

Tendo em vista a redução da morbimortalidade materna e infantil, em 2011, o Ministério da Saúde do governo federal instituiu o plano Rede Cegonha no âmbito do Sistema Único de Saúde (SUS). A Rede Cegonha tem como objetivo organizar a atenção materno-infantil no Brasil, assegurando que toda mulher tenha direito ao planejamento reprodutivo, bem como a assistência integral no pré-natal, durante o parto e também no puerpério (pós-natal), além de garantir o pleno crescimento e desenvolvimento da criança (5).

No ano de 2012, o Paraná implantou a Rede Mãe Paranaense, seguindo os mesmos princípios da Rede Cegonha. Uma das estratégias do programa é a identificação e prevenção de condições de risco através da classificação da gestante e da criança em três grupos: alto risco, risco intermediário e abaixo risco (6).

De acordo com o Sistema de In-
O Brasil conseguiu reduzir a mortalidade infantil entre 1990 e 2015, uma das metas dos Objetivos de Desenvolvimento do Milênio (ODM). Entretanto, no ano de 2016, a taxa de mortalidade infantil apresentou um crescimento, fato que não acontecia há 26 anos formação de Mortalidade da Secretária de Saúde do estado do Paraná, no ano de 2010, antes da implantação da Rede Mãe Paranaense, o coeficiente de mortalidade infantil era de 12,65/1.000 nascidos vivos. Já no ano de 2014, após a implantação do programa, a taxa sofreu uma pequena redução, para 11,65/1.000 nascidos vivos. Em 2019 o número de óbitos registrado foi de 10,62/1.000 nascidos vivos. Esta redução não foi significativa, visto que, grande parte dos óbitos foram por causas evitáveis (7).

Considerando o fato de que o Programa visa a continuidade do acompanhamento após o nascimento da criança, para que se reduza a taxa de mortalidade infantil, principalmente de causas preveníveis, é indiscutível a necessidade de estudos sobre os motivos que levam as mães a abandonarem o acompanhamento no Programa Rede Mãe Paranaense (7).

Devido ao cenário de fragilidade, torna-se importante a vinculação de mães e bebês em políticas públicas de saúde que transformem suas condições de vida, após o nascimento e propicie a prevenção de condições de risco em que o binômio mãe-filho se encontram. Embora exista uma política pública de atendimento específica para os casos de mães e bebês de risco, ocorre o abandono do acompanhamento da criança no primeiro mês de vida no Programa Rede Mãe Paranaense (7-4).

Assim, o estudo justifica-se por buscar a percepção das mães para o abandono no seguimento da criança recém-nascida, de risco, apesar da existência de um programa de atenção à saúde para esse público específico. Dessa forma, este estudo teve por objetivo identificar os motivos que levam as mães de bebês de risco a abandonarem o acompanhamento no Ambulatório de Alto Risco, ao longo do primeiro ano de vida. 


\section{MÉTODO}

Estudo descritivo e exploratório, de natureza qualitativa realizado com 16 mães de bebês que abandonaram o acompanhamento dos bebês no Ambulatório de Alto Risco. Utilizou-se a diretriz COREQ (Consolidated Criteria for Reporting Qualitative Research) como direcionamento para sua produção (8).

Os participantes do estudo foram mães de crianças com idades entre até 12 meses de idade cadastradas no Programa Mãe Paranaense, no Ambulatório de Alto Risco referência para uma Regional de Saúde do Estado do Paraná. Os critérios de inclusão foram crianças estratificadas como alto risco segundo os critérios da Rede Mãe Paranaense e, encaminhadas para o acompanhamento no ambulatório especializado no segundo semestre do ano de 2019, e o informante ser o cuidador principal da criança. As mães de bebês que foram a óbito no período foram excluídas do estudo.

À saber, crianças de alto risco são aquelas apresentam fatores de risco relacionados a: afecções Perinatais (Prematuridade $\leq 34$ semanas de idade gestacional; baixo peso ao nascer $<2.000$; asfixia perinatal- apgar $<7$ no $5^{\circ}$ minuto), hiperbilirrubinemia com exsanguineotransfusão, más-formações congênitas/ cromossopatias/ doenças genéticas, triagem neonatal positiva, doenças de transmissão vertical confirmadas, desnutrição grave, obesidade, atraso do desenvolvimento neuropsicomotor e ntercorrências repetidas com repercussão clínica(6).

Do total de 75 crianças cadastradas (2 gemelares), 26 mães não foram localizadas por alteração do contato telefônico. Das 48 mães localizadas, 32 recusaram-se em participar do estudo, compondo-se a amostra com 16 mães.

A coleta de dados ocorreu nos meses de agosto a outubro de 2020, por meio de agendamento prévio para aplicação de entrevistas semiestruturadas com perguntas que versam acerca dos motivos que levam as mães de bebês de risco a abandonarem o acompanhamento no Ambulatório de Alto Risco, ao longo do primeiro ano de vida.

Após a leitura do Termo de Consentimento Livre e Esclarecido para as mães e do seu consentimento em participar do estudo, as entrevistas foram gravadas e, posteriormente transcritas na íntegra. O material transcrito foi submetido à análise de conteúdo, modalidade temática (9), sendo assim emergiram duas categorias intituladas: Abandono do acompanhamento infantil de alto risco: desconhecimento atrelado a falta de apoio social; Ansiedade frente ao desconhecido: vivência das mães após o nascimento.

Para a realização da presente pesquisa, foram seguidos todos os preceitos éticos e legais contidos nas normas regulamentadoras determinadas pelas Resoluções 466/2012, 510/2016 do Conselho Nacional de Saúde, e que estabelecem as diretrizes éticas para a realização de estudos com seres humanos. O estudo obteve o parecer de apreciação ética pelo Comitê Permanente de Ética em Pesquisa Envolvendo Seres Humanos da Universidade Estadual de Maringá sob o registro $\mathrm{n}^{\circ} 3.766 .436$, CAAE: 24906719.9.0000.0104.

Com o propósito de preservar o anonimato na apresentação dos resultados, os extratos dos relatos dos participantes foram identificados pela letra " $M$ " de Mãe, um número que informa a ordem da realização da entrevista.

\section{RESULTADOS}

Foram entrevistadas 16 mães de crianças encaminhadas ao Ambulatório de Alto Risco referência para uma Regional de Saúde do Estado do Paraná. Dentre as participantes do estudo, 12 pertenciam ao grupo de abandono do acompanhamento, duas do grupo de adesão ao acompanhamento e duas eram do grupo de não adesão. Nove delas eram residentes na cidade de Maringá e sete eram de cidades próximas, pertencentes à $15^{\mathrm{a}}$ Regional de Saúde do Paraná.

Dos prontuários analisados, sete $(43,75 \%)$ crianças eram do sexo feminino e outras nove $(56,25 \%)$ eram do sexo masculino. Os motivos de encaminhamento foram principalmente por prematuridade, correspondendo a 43,75\% e doenças de transmissão vertical (sífilis, toxoplasmose e hepatite B), com cerca de $37,5 \%$, conforme mostra a tabela 1 .

Em relação ao tempo de acompanhamento no ambulatório, 50\% (8) das crianças foram acompanhadas por quatro meses ou menos, $18,75 \%$ (3) de cinco a oito meses e 31,25\% (5) de nove meses ou mais. A média do número de consultas por criança foi de 2,37.

Do processo de análise dos dados emergiram duas categorias temáticas que serão descritas a seguir.

Abandono do acompanhamento infantil de alto risco: desconhecimento atrelado a falta de apoio social

Verificou-se que algumas mães não possuíam conhecimento sobre o protocolo de acompanhamento previsto pela Rede Mãe Paranaense para as crianças estratificadas como de alto risco, bem como os motivos da inclusão do seu fiIho no programa.

Se tivessem me explicado corretamente, tudo certinho, eu tinha ido. Ainda mais sendo filho, a gente quer sempre o bem, a gente faz o que pode e se me explicassem o que era o melhor a fazer, por quê, entendeu? Com certeza teria ido, tudo por ele. (M1)

A moça que deu alta para gente, é, ela só falou assim, que ele seria acompanhado só para ter certeza de que estava tudo ok, mas que ele estava bem. (M2)

Acredita-se que a orientação da 


\begin{tabular}{|c|c|c|}
\hline $\begin{array}{c}\text { Motivos de } \\
\text { encaminhamento }\end{array}$ & $\mathrm{n}$ & $\%$ \\
\hline Prematuridade & 7 & 43,75 \\
\hline $\begin{array}{c}\text { Doenças de transmissão } \\
\text { vertical }\end{array}$ & 6 & 37,50 \\
\hline Nefropatias & 1 & 6,25 \\
\hline Pneumonias & 1 & 6,25 \\
\hline Síndromes & 1 & 6,25 \\
\hline
\end{tabular}

Fonte: dados da pesquisa (2020).

mãe seja importante para a adesão ao acompanhamento. Entretanto, existem outros motivos que influenciaram no abandono e, esses não estavam relacionadas apenas a fatores maternos, visto que as condições dos serviços também influenciaram diretamente na situação da mãe e da criança nas consultas.

Quando questionadas sobre o motivo do abandono ao acompanhamento as justificativas foram: incompatibilidade de horário diante da agenda do serviço; falta de flexibilidade do serviço de saúde; dificuldade de acesso ao serviço; percepção de que a criança estava com bom desenvolvimento; questões pessoais e a pandemia do COVID-19.

Eu tentei marcar várias vezes a consulta novamente, mas não conseguia, porque eles não queriam marcar por telefone e como eu trabalhava, eu não conseguia ficar faltando no serviço para poder marcar a consulta. (M1)

Por causa do serviço mesmo né, porque não tinha como eu ficar levando, porque eu não, não sou de Maringá, sou de Paiçandu né. (M10)

O desenvolvimento dela estava muito bom... ela não tinha mais problema nenhum, tanto é que ela ficou sem nenhuma sequela né, por causa da prematuridade. (M14)

Teve algumas vezes que foi por conta da minha consulta e outra foi porque ele estava gripado, daí eu não levei e também não avisei. (M13)

Eu só parei de ir mesmo por conta do coronavírus, senão eu estaria indo nas consultas certinho, como sempre fui. (M16)

Como apresentado nas representações dos discursos acima, o conhecimento das mães acerca da condição do filho é decisivo para o acompanhamento infantil, pois é este que trará o senso da necessidade, diminuindo as faltas.

Ansiedade frente ao desconhecido: vivência das mães após o nascimento

O pós-parto é um momento delicado para algumas mães. Um misto de sentimentos foi gerado no instante em que estas tomam conhecimento da necessidade do acompanhamento e até mesmo durante as consultas. Parte das mães tiveram medo em relação a resultados de exames/bem-estar da criança. O medo e a preocupação foram sentimentos negativos, inevitáveis, iniciais e comuns à maioria das mães durante as primeiras consultas.

Eu tinha medo de quando eu chegasse lá na consulta, o resultado do exame não fosse bom, sabe? Porém, eu sempre chegava e os exames eram bons. Eles, assim, aquela angústia que estava dentro de mim, os médicos, na consulta né, o pessoal que atendia ela, sempre me acalmava, dando o resultado bom, entendeu? (M4)

Os resultados de exames e condutas médicas foram condições que também geraram ansiedade em uma das mães, visto que são situações incertas que podem gerar algum dano ao bebê.

"Cada consulta que eu ia, eu ficava ansiosa, nervosa com que a doutora ia me falar, com os resultados dos exames que ela fazia, mas no final sempre ficava aliviada (M6)"

Apesar de existirem relatos de sentimentos negativos, muitas mães expressaram também sentimentos positivos em relação as consultas. Os sentimentos de confiança, segurança e satisfação foram relatados pela maior parte das entrevistadas. Os profissionais do Ambulatório de Alto Risco foram diversas vezes exaltados pelos serviços $\mathrm{e}$ atendimento oferecidos.

Eu me senti bem, eu me senti segura, senti que eu não estava sozinha para cuidar dela né, que qualquer, qualquer problema que talvez surgisse depois, eu não ia ter que correr sozinha com ela ou descobrir o que ela tinha sozinha. (M3)

A pediatra e as estagiárias dela me passavam bastante confiança e não tenho o que reclamar do atendimento do ambulatório, sempre fui muito bem tratada e eu só tinha um pouco de medo, por causa dessas alterações nos exames dele. (M8)

Apesar dos relatos da percepção benéfica do acompanhamento infantil de alto risco, a falta no acompanhamento e o consequente abandono parecem inevitáveis. Neste interim ressalta-se a importância da presença do profissional de saúde na adesão ao programa. Foi observado que metade das mães relataram alguma deficiência da busca ativa como fator importante para o abandono.

Eu não recebi nenhuma ligação, 
nenhuma mensagem para remarcar, então eu acho que eles poderiam ter me ligado para, é, falar se eles ainda estavam atendendo mesmo com o começo da pandemia. (M7)

Se eles tivessem me ligado, falando né, da importância de acompanhar, eu acho que eu deveria ter continuado né. (M10)

A busca ativa das crianças faltosas, demonstrou-se fragilizada corroborando para o aumento de casos para o abandono do acompanhamento infantil.

\section{DISCUSSÃO}

O maior número de crianças do sexo masculino encaminhadas ao ambulatório, coaduna com o menor número dos nascimentos de crianças do sexo feminino em todo o país, que no ano de 2019 apresentava uma proporção de $2,6 \%$ a mais de nascimento de meninos no período analisado (10).

Observou-se que a média de consultas por criança foi de 2,37, resultado inferior ao preconizado pela Linha Guia da Rede Mãe Paranaense. O programa define que a criança estratificada como Alto Risco deve realizar acompanhamento até os 12 meses de vida e com no mínimo 4 consultas multiprofissionais (6).

De acordo com os relatos, os motivos de abandono ao acompanhamento dos filhos foram diversos, inclusive o fato de que algumas mães não tinham conhecimento acerca do acompanhamento no Ambulatório de Alto Risco. O Programa prevê orientações às mães sobre o motivo do encaminhamento, a importância das consultas e os riscos que a criança pode enfrentar sem devida assistência profissional. No entanto, os profissionais do serviço são os grandes responsáveis por essa orientação, não apenas no momento da alta hos- pitalar, mas em todos os retornos ambulatoriais. A educação em saúde deve ser promovida, para que as famílias desenvolvam um olhar mais crítico sobre a saúde das crianças (7).

A ansiedade frente a doença do fiIho causa, em geral apreensão nos pais. Muitas mães ao serem informadas sobre o diagnóstico do filho apresentam tristeza e culpa por poderem ter sido responsáveis por sua doença e temendo suas complicações. A falta de um diagnóstico inicial preciso pode levar as mães a temerem os piores cenários para um desfecho dos filhos. A preocupação e o desespero são sentimentos frequentes e a espera por um diagnóstico gera nas mães uma experiência de impotência e extrema angústia para mães de filhos doentes (11).

As aceitações de novas situações podem ser definidas a partir da sensibilidade do profissional de saúde que aborda o diagnóstico, assim como todo o apoio multiprofissional que os familiares. A possibilidade de encontrar profissionais humanizados, que, mesmo diante das dúvidas, buscam acalentar, dar esperanças e confortar as mães ajudam na condução do processo de acompanhamento de saúde do filho. Essa conduta representa um chamado atentivo para a efetivação da política pública de humanização de atenção à saúde (12).

Outros motivos foram citados pelas mães que justificavam o abandono do acompanhamento do filho. A falta de flexibilidade dos serviços de saúde e a dificuldade de acesso são fatores muito mencionados pelas participantes. Os horários de atendimento dos serviços de saúde coincidem com os horários comerciais de trabalho de muitas mães, fazendo com que a falta de flexibilidade para o atendimento, fossem responsáveis pelo abandono (13).

A inserção da mulher no mercado de trabalho se deve as guerras que marcaram a história da humanidade, em que os homens tinham que ir para 
as batalhas e as mulheres assumiam os negócios da família. Desse modo, a partir desse marco, com o passar do tempo, as mulheres progressivamente cresceram e conquistaram o seu espaço no mercado de trabalho. No entanto, além de sustentarem a casa, muitas mulheres ainda possuem casa e filhos para cuidarem. É necessário que os serviços de saúde entendam a realidade dos seus usuários para que possam readequar o atendimento oferecido, bem como a infraestrutura e garantir assim a qualidade e integralidade do serviço em saúde (13).

Além disso, a percepção materna acerca do crescimento e desenvolvimento adequado do filho, em função da ausência da doença e por considerar que a criança estava saudável, foi um dos fatores mais relatados. Essa percepção é influenciada pela questão sociocultural e pelos recursos disponíveis, que influenciam no significado do que a mãe atribui como necessário ou não para o filho. Entende-se que os saberes e práticas precisam ser compartilhados entre profissionais e famílias, reconhecendo as suas diferenças e contribuições para o atendimento às necessidades das crianças(14).

A pandemia do COVID-19 foi outro fator que teve grande influência no abandono do acompanhamento. As mães relatarem que no início da pandemia tinham medo em levar as crianças nos hospitais, visto que não se tinha muitas informações acerca da doença. Em função da doença, muitas crianças deixaram de ser acompanhadas, sendo expostas a situações de risco em função da sua condição de fragilidade (15).

A COVID-19 é uma doença infecciosa, altamente transmissível, causada pela SARSCoV-2, patógeno classificado como uma nova variante do gênero Beta Coronavírus. Foram presenciados inúmeros casos, que logo espalharam-se rapidamente pelo mundo, chegando ao Brasil em fevereiro de 2020, onde diversas medidas foram implantadas para conter o avanço da doença. A transmissão ocorre pelo contato direto com secreções ou gotículas de uma pessoa infectada, formadas durante a fala, tosse ou espirro. As manifestações clínicas podem variar, podendo ser leve, assintomática ou grave, este por sua vez, podendo ocorrer com maior frequência

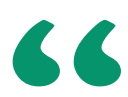

A pandemia do COVID-19 foi outro fator que teve grande influência no abandono do acompanhamento. As mães relatarem que no início da pandemia tinham medo em levar as crianças nos hospitais, visto que não se tinha muitas informações acerca da doença

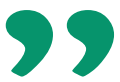

em idosos, crianças e grupos de risco, como indivíduos imunodeprimidos. Portanto, devido a recomendação do isolamento social ao combate da transmissão do vírus, as poucas informações acerca da doença e o medo frente ao desconhecido, muitas mães optaram por não levar os seus filhos nas consultas, ocasionando a descontinuidade no tratamento (15-16).

A descontinuidade é prevista, e portanto, a busca ativa é parte importante do Programa Rede Mãe Paranaense, contudo esta mostrou-se bastante deficiente no presente estudo, principalmente após o início da pandemia. Seu objetivo principal é identificar as necessidades de saúde de indivíduos de um determinado território, para além da demanda espontânea, garantindo a integralidade do cuidado. Essa ação é fundamental para o pleno crescimento e desenvolvimento da criança. Através desta ação, é possível localizar as crianças faltosas, traze-las e mantê-las até a alta das consultas. Além disso, a busca ativa permite entender as condições socioeconômicas e territoriais que podem levar ao abandono (17).

O abandono do acompanhamento das crianças consideradas mais frágeis pode levar a graves riscos à saúde, mesmo que não apresentem sintomas que possam ser observados pelas mães. Desse modo, os serviços de saúde devem buscar entender melhor as características da população, para que possam adequar os serviços de saúde a mesma (18).

Destaca-se como limitação do presente estudo, o fato de ter sido realizado em um único ambulatório de alto risco, o que pode ter circunscrito seus resultados à perspectiva dos participantes que vivenciam a experiência do abandono. Apesar disso, acredita-se que os achados desse estudo possam subsidiar reflexões acerca dos elementos envolvidos no processo do acompanhamento dos bebês de alto risco apontando os principais aspectos a serem considerados na assistência desse público.

\section{CONCLUSÃO}

O estudo permitiu a identificação dos motivos referente aos aspectos maternos, familiares, sociais e características dos serviços de saúde que podem 
influenciar no abandono do acompanhamento infantil. Através do conhecimento desses fatores, é possível definir novas estratégias e ações para suprir as necessidades da população.
Ressalta-se a importância dos profissionais no acolhimento dos usuários e permanência destes no programa. Os profissionais possuem grande papel na adesão ao serviço e devem estar sempre buscando conhecimento e capacitação para receberem as mães e as crianças, criando vínculos e, as motivando cada vez mais a irem nas consultas.

\section{Referências}

1. França $E B$ et al. Principais causas da mortalidade na infância no Brasil, em 1990 e 2015: estimativas do estudo de Carga Global de Doença. Rev Bras Epidemiol. [Internet]. 2017[citado 2020 nov 28];20(1):46-60. Disponível em: https://doi.org/10.1590/1980-5497201700050005

2. United Nations Inter-agency Group for Child Mortality Estimation (UN IGME). Levels \& Trends in Child Mortality: Report 2019, Estimates developed by the United Nations Interagency Group for Child Mortality Estimation. United Nations Children's Fund, New York, 2019. Disponível em: https://childmortality.org/wp-content/uploads/2019/10/UN-IGMEChild-Mortality-Report-2019.pdf.

3. Instituto Brasileiro de Geografia e Estatística. Projeção da população do Brasil por sexo e idade para o período 2010 - 2060. Rio de Janeiro, IBGE, 2018. Disponível em: https://www.ibge.gov.br/estatisticas/sociais/populacao/9109-projecao-dapopulacao.html?=\&t=0-que-e.

4. Netto $A$, da Silva R, Santos M, Tacla M, Caldeira S, Brischiliari S. Mortalidade Infantil: Avaliação do Programa Rede Mãe Paranaense em Regional de Saúde do Paraná. Cogitare Enferm. [Internet]. 2017 jan/ mar[citado 2020 nov 28];22(1):01-08. Disponível em: .doi.org/10.5380/ ce.v22i1.47634

5. Brasil. Ministério da Saúde. Gabinete do Ministro. Portaria n 1.459, de 24 de junho de 2011. Institui, no âmbito do Sistema Único de Saúde - SUS - a Rede Cegonha. Disponível em:

https://bvsms.saude.gov.br/bvs/saudelegis/gm/2011/ prt1459_24_06_2011.html.

6. Paraná. Linha Guia Rede Mãe Paranaense. Secretaria de estado da saú-

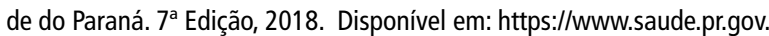
br/sites/default/arquivos_restritos/files/documento/2020-09/LinhaGuiaMaeParanaense_2018.pdf

7. Santos DR, Viera CS, Guimarães ATB, Toso BRGO, Ferrari RAP. Avaliação da eficácia do Programa Rede Mãe Paranaense. Saúde em Debate. [Internet]. 2020[citado 2020 nov 28];44(124):70-85. Disponível em: https:// doi.org/10.1590/0103-1104202012405

8. Tong A, Sainsbury P, Craig J. Consolidated criteria for reporting qualitative research (COREQ): A 32-item checklist for interviews and focus groups. Int J Qual Heal Care. [Internet]. 2007[citado 2020 nov 28];19(6):349-57. Disponível em: https://doi.org/10.1093/intqhc/mzm042

9. Bardin L. Analise de Conteúdo. São Paulo: Ed. Revista Ampliada; 2016.
10. Brasil. Sistema de Informações de Nascidos Vivos. Ministério da Saúde: Brasília, 2020. Disponível em: http://tabnet.datasus.gov.br/cgi/tabcgi. exe?sinasc/cnv/nvpr.def.

11. Costa LD, Hoesel TC, Teixeira GT, Trevisan MG, Backes MTS, Santos EKA. Percepções de gestantes internadas em um serviço de referência em alto risco. Rev Min Enferm. [Internet]. 2019[citado 2020 nov 28];23:e-1199. Disponível em: http://www.dx.doi.org/10.5935/1415-2762.20190047

12. Oliveira MC, Moreira RCR, Lima MM, Melo RO. Vivências de mães que tiveram filhos com microcefalia. Rev Baiana Enferm. [Internet]. 2018[citado 2020 nov 28];32:e26350. Disponível em: https://doi.org/10.18471/ rbe.v32.26350

13. Cardoso VEPS, da Silva Junior AJ, Bonatti AF, Santos GWS, Ribeiro TAN. The Partner's Involvement in the Prenatal Routine Through the Pregnant Women Perspective. R pesq cuid fundam online. [Internet]. 2018[citado 2020 nov 28];10(3):856-62. Disponível em: https://doi.org/10.9789/21755361.2018.v10i3.856-862

14. Diniz IA, Guimarães BR, Silva JB, Tavares TS, Duarte ED. Descontinuidade do seguimento ambulatorial de crianças de risco: perspectiva das mães. Esc Anna Nery. [Internet]. 2019[citado 2020 nov 28];23(2):e20180248. Disponível em: https://doi.org/10.1590/2177-9465-EAN-2018-0248

15. Albuquerque LP, Silva RB, Araújo RMS. COVID-19: origem, patogênese, transmissão, aspectos clínicos e atuais estratégias terapêuticas. Rev Pre Infec e Saúde. [Internet]. 2020 [citado 2020 nov 28];6:10432. Disponível em: : https://doi.org/10.26694/repis.v6i0.10432

16. Brasil. Protocolo de Manejo Clínico do Coronavírus (CoVId-19) na Atenção Primária à Saúde. Versão 9. Ministério da Saúde: Brasília, 2020.

Disponivel em: https://www.unasus.gov.br/especial/covid19/pdf/37.

17. Tavares MNM, Silva Filho JA, Silva CRL, Pinto AGA. Consulta de enfermagem em puericultura na estratégia saúde da famí $\neg$ lia: revisão integrativa. Nursing. [Internet]. 2019[citado 2020 nov 28];22(256):3144-9. Disponível em: https://doi.org/10.36489/nursing.2019v22i256p3144-3149

18. Shibukawa BMC, Rissi GP, Uema RTB, Merino M de FGL, Hoffmann Cheffer M, Higarashi IH. Comportamento faltoso em serviços de saúde materno-infantil: revisão integrativa da literatura. Ver Eletr Enferm. [Internet]. 2021 [citado 2020 nov 28];23. Disponível em: https://revistas.ufg. br/fen/article/view/65051 